\title{
El lugar del adulto mayor en una educación orientada a la trasformación social y desarrollo humano*
}

\author{
The elderly adult place in an education oriented \\ to social transformation and human development
}

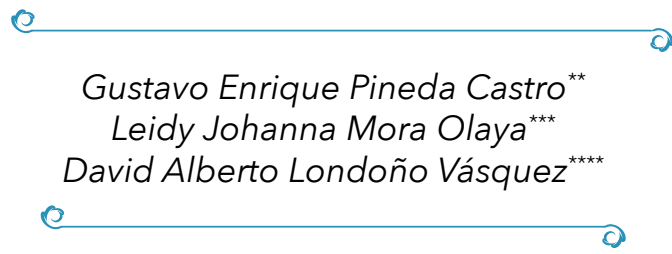

Recibido: 15.04.2020 • Arbitrado: 05.05.2020 •

Aprobado: 26.05.2020

* Este artículo de revisión se desprende de la tesis Condiciones personales, sociales y familiares que instan a los adultos mayores a ingresar a la educación formal: La experiencia en los Ciclos Lectivos Integrados (CLEI) del Municipio de Sabaneta (Antioquia), la cual fue aprobada como requisito de grado en la Maestría en Educación y Desarrollo Humano de la Universidad de Manizales-Cinde en el 2020.

* Magíster en Educación y Desarrollo Humano. ORCID: https://orcid. org/0000-0001-6268-0724, Correo electrónico: gustavopinedacastro@ hotmail.com

*** Magíster en Educación y Desarrollo Humano. ORCID: https://orcid. org/0000-0003-0980-3056, Correo electrónico: leijomora@hotmail.com

**** Doctor en Ciencias Sociales, Niñez y Juventud, miembro del grupo de investigación en Psicología Aplicada y Sociedad de la Institución Universitaria de Envigado, Docente de Tiempo Completo de la Institución Universitaria de Envigado. Researcher ID: F-8907-2013. ORCID: https://orcid. org/0000-0003-1110-7930. Correo electrónico: dalondono@correo.iue.edu.co

\section{Resumen}

Objetivo: Este artículo aborda el tema del desarrollo humano a partir de la educación sin limitaciones etarias, donde el adulto mayor puede seguir (re)construyéndose a través de su participación en procesos educativos formales.

Metodología: Selección de literatura a partir de cinco descriptores previamente definidos, en español, inglés y portugués, a través de Google Académico, teniendo en cuenta los criterios de visibilización e impacto.

Resultados: Para ello, se resalta la educación desde una perspectiva crítica, la cual permite concebirla como una de las vías para la transformación social, destacando la posibilidad de vencer obstáculos, sobreponerse a las dificultades y avanzar a nivel personal, familiar y académico, tomando como pretexto a los adultos mayores que integran los programas de educación flexible en Colombia. 
Conclusiones: La formación se convierte en una opción para afianzar el tejido social, donde la alfabetización juega un papel vital en la búsqueda de la equidad y el reconocimiento del adulto mayor y propende por el desarrollo de habilidades que pueden ser requeridas en el proceso de formación escolar andragógico.

Palabras clave: Adulto mayor, andragogía, educación, transformación social.

\section{Abstract}

Objective: This paper addresses the issue of human development from education without age limitations, where the elderlies can continue to (re)build themselves through their participation in formal educational processes.

Methodology: Selection of corpus from five previously defined descriptors, in Spanish, English and Portuguese, through Google Scholar, taking into account the visibility and impact criteria.

Results: For this, education is highlighted from a critical perspective, which allows it to be conceived as one of the avenues for social transformation, highlighting the possibility of overcoming obstacles, overcoming difficulties and advancing on a personal, family and academic level, taking as a pretext for older adults who are part of flexible education programs in Colombia.

Conclusions: Training becomes an option to strengthen the social fabric, where literacy plays a vital role in the search for equity and the recognition of the elderly and tends to develop skills that may be required in the process of andragogic school training.

Key words: Andragogy, elderly, education, social transformation.

\section{Introducción}

Históricamente, la sociedad ha tenido diferentes roles con respecto al individuo; puesto que, parte de su función es la de propender por la interacción, la regulación, la formación y la participación (Lahire, 2007). Allí, la familia, la escuela y los amigos han servido como escenarios de socialización (Simkin y Becerra, 2013), permitiendo que se construyan formas de consolidación de una sociedad (Allman, 2001).

La estrecha relación que esta ha tenido con la formación del individuo, sugiere un alto impacto frente a la educación y las diversas concepciones de la misma (Crichlow, 2013). Se ha pasado en los últimos años de una sociedad de la información a una del conocimiento también en la formación (Anderson, 2008). En este orden de ideas, se entiende una educación orientada no 
tanto a la trasmisión de la información (Almiron y Porro, 2014), que está siempre al alcance de todos en la sociedad contemporánea, sino, más bien, al desarrollo de las habilidades blandas y habilidades para la vida (López, Dominguez, Álvarez y Jaén, 2011), dentro de un marco de transformación social y desarrollo humano (Alkire, 2002).

Por otro lado, se trata de una educación continuada a lo largo de la vida, donde los limites etarios tradicionales dejan de ser vigentes y emerge una amplia población de adultos mayores que anteriormente eran considerados por fuera de su alcance.

En otras palabras, la educación debe ser entendida como una de las vías para la trasformación social (Tuckett, 2015) y reconociendo las situaciones problemáticas que afrontan quienes desean ingresar o continuar su educación, tales como: escasos recursos económicos (Allman, 2001), poco apoyo familiar (Villalobos, Flórez y Londoño, 2017b), limitaciones de tiempo (Díez y Flecha, 2010) y jornadas laborales extensas (Bracher, 2006), es factible afirmar que, tanto desde el sector público, como desde la misma sociedad, es posible generar estrategias que permitan minimizar la deserción académica (Henao y Londoño, 2017) y brindar garantías de permanencia en las instituciones educativas para adultos mayores (Medrano, Galleano, Galera y del Valle Fernández, 2010), además de motivar a la comunidad a continuar estudiando sin importar el nivel académico que se ostente (Tejada y Ferrández, 2012).

En varios municipios de Colombia, se vienen implementando los CLEI para adultos mayores. Este programa refiere a unidades curriculares equivalentes a determinados grados de educación formal regular, constituidos por objetivos y contenidos pertinentes, debidamente seleccionados e integrados secuencialmente con el objetivo de que los participantes alcancen los logros establecidos (MEN, 2018).

Vale mencionar que este programa propende por la educación del adulto mayor desde la mirada del desarrollo humano (Mogollón, 2012), el trabajo cooperativo (Ubaldo, 2009) y la adecuada comunicación (Martínez de Morentin, 2006), situación que permite que los adultos mayores sean vistos de otra manera y que representa una invitación general a la academia.

Este artículo se orienta desde una mirada distante a la educación tradicional, puesto que plantea la posibilidad de trabajar de acuerdo con las realidades de los sujetos (Bradbury, 2013) y el territorio que ocupan (Calvo, 2012); es decir, una educación desde el desarrollo humano y la atención a las circunstancias prácticas de la vida diaria (Tuckett, 2015), la cual requiere 
de parámetros que permitan generar identidad, arraigo y compromiso por mejorar las condiciones en sus localidades (Crichlow, 2013), fortaleciendo así sus lazos con sus antepasados, familia, vecinos y comunidad en general (Villalobos, Flórez y Londoño, 2017a).

Además, esta apuesta permite entender la andragogía (Ubaldo, 2009) como una postura pedagógica y metodológica que debe fundamentarse en las necesidades de este grupo etario (Caraballo, 2007), orientándose al objetivo de aportar a una mejor calidad de vida en esta etapa evolutiva (Ozuah, 2016).

De allí que esta revisión se centró, precisamente, en identificar algunas ideas disponibles en literatura sobre la inclusión del adulto mayor en la educación, su efecto a nivel del desarrollo humano en esta etapa de la vida y los aspectos distintivos que debe tener el abordaje educativo del adulto mayor.

\section{Metodología}

Esta revisión se desprende de una tesis de Maestría en Educación y Desarrollo Humano aprobada en el 2020. La literatura revisada se seleccionó a partir de cinco descriptores previamente identificados relacionados con el tema (Ramírez, 2020). Estos fueron: adulto mayor y educación; andragogía y desarrollo humano; desarrollo humano y familia; educación y transformación social del adulto; familia y educación del adulto.

La búsqueda se realizó en español, inglés y portugués. Se seleccionaron los 10 textos con mayor citación en Google Académico por descriptor, teniendo en cuenta los conceptos de visibilización e impacto (Pérez, García y Aguaded, 2018).

El análisis permitió la construcción de 4 categorías, las cuales fueron:

- La educación y el desarrollo humano

- La educación del adulto mayor como una opción para afianzar el tejido social

- La alfabetización como garante de equidad y reconocimiento

- Algunos efectos del a educación en el adulto mayor

A continuación, se presenta la revisión realizada de forma cualitativa (Guirao, 2015), desde una perspectiva hermenéutico-comprensiva (Uribe, Ramírez y Londoño, 2020) y mediada por una matriz de agrupación conceptual (Ramírez, 2020). 


\section{Desarrollo del tema}

\section{La educación y el desarrollo humano}

La educación, entonces, es un medio de avance mediante el cual el ser humano adquiere habilidades (Rogers, 2013) y enriquece la cultura para desarrollarse (Knowles, Holton \& Swanson, 2014), afrontar las dificultades (Barraza, 2011), expandir la creatividad promoviendo la evolución independiente a nivel cultural, social y personal (Giroux, 2010), pues, "la educación verdadera es praxis, reflexión y acción del hombre sobre el mundo para transformarlo" (Freire, 1986, p.1).

Entre tanto, enriquece al hombre de conocimientos e influye en su avance y progreso fortaleciendo los valores, el espíritu y el saber (Castillo y Cardona, 2019), alcanzándose un bienestar social y un crecimiento económico (Quintero, Valencia y Alvarez, 2019); en otras palabras, gracias a la educación "se accede a niveles más adecuados de empleo, se amplían las oportunidades y se fortalecen las relaciones de las sociedades procurando el impulso y avance de la ciencia, la tecnología y la innovación" (Narro, Martuscelli, \& Barzana, 2012 p.1), en consecuencia con las posibilidades del entorno y los planes de vida trazados.

Por tanto, es posible indicar que la educación es un fenómeno complejo que está inmerso en prácticas personales, sociales, culturales e históricas muy amplias (Batalloso, 2006). De allí que, cada acto educativo esté influenciado por dichas prácticas, en consecuencia, la educación necesitará de un razonamiento cualitativo diferente, con el fin de evitar las deformaciones y obstáculos que impiden el desarrollo pleno de la persona (Suter, 2011).

Ahora bien, en cuanto a la educación orientada al desarrollo humano, se debe enfatizar en que esta supone un proceso que apunta a conocer las realidades del estudiante, lo que lo rodea, sus intereses y actividades (Knowles, et al., 2014), partiendo de entender las necesidades y deseos de cada uno de los participantes (Marrero, 2004), para esto es crucial la comunicación asertiva y efectiva (Martínez de Moretin, 2006). Puesto que, un adecuado proceso educativo para el desarrollo humano debe partir del diálogo con la cultura, promoviendo la convivencia, la participación, la inclusión y el sentido de pertenencia que satisface la necesidad de identidad (Ospina, 2008).

De otro lado, es significativo analizar las relaciones y comunicaciones que se dan entre el educador y los educandos, relación que debe constituir un ejercicio en doble vía, es decir, no solo desde la transmisión de conocimientos, sino, además, de un interés de comprensión y entendimiento. 


\section{La educación del adulto mayor como una opción para afianzar el tejido social}

Se habla, entonces, de una relación social presente en el devenir cotidiano, de personas que participan en las actividades de la vida colectiva (Rodríguez, 2018) que consiste en lograr que la humanidad pueda dirigir y estructurar su propio desarrollo (Nussbaumn y Mosquera, 2012), contribuyendo a comprender el mundo y los demás (Rogers, 2013). Por otro lado, es común, en el imaginario que se asiste a una institución educativa por una certificación o un conocimiento general (Tejada y Ferrández, 2012); no obstante, se puede pensar que por algo más, por compromiso social o familiar (Díez y Flecha, 2010), por dignidad (Bracher, 2006) o por entender aspectos prácticos de la vida diaria, como firmar sin temor y recibir cualquier tipo de documentación con la seguridad de comprender lo que quiere ser transmitido por otros (Franco, Londoño y Restrepo, 2017). Lo anterior sirve de base para el desarrollo humano en el que se crea un medio ambiente con una realidad llena de posibilidades para vivir productivamente conforme a sus intereses y necesidades. De allí que:

(...) un proceso por el cual las personas que ya no asisten a la escuela de tiempo completo (...) prosiguen actividades secuenciales y organizadas con la intención consciente de producir cambios en información, conocimientos, comprensión, destrezas, apreciación y actitudes (...) el verdadero propósito de la educación de adultos es ayudar a la gente en un mundo de rápidos cambios y a construir sus fines realmente en términos adecuados, y ayudar a las personas a comprender su problema, descubrir los recursos a su alcance, y encontrar el camino a la solución de sus problemas y el alcance de sus fines dentro de situaciones corrientes. (Bastista, 2006, p.57).

Ahora bien, de lo que se trata es de que el adulto mayor en cuanto a la educación, desarrolle los medios necesarios para alcanzar los saberes que incrementen metas (Caraballo, 2007) y se instruya porque quiere (Ubaldo, 2009), aprenda a resolver situaciones de conflicto a través de una participación flexible (Ospina, 2008), logrando una motivación de la vida diaria en la que pueden encaminar sus pasos a la innovación y la imaginación (Allman, 2001), en una educación que guía de manera colaborativa frente a unas necesidades que dependiendo del tipo de contenido sea asumida desde una metodología flexible (Mogollón, 2012), porque el derecho a la educación más que contribuir a una cultura del envejecimiento, representa una oportunidad 
de participación social y reafirmación de habilidades y saberes (Suter, 2011) que elevan la calidad de vida viéndose reflejada en bienestar y salud, no siendo sometida a límites de edad (Tuckett, 2015), sino de aquellas personas que desean mejorar sus aptitudes, enriquecer sus conocimientos y afianzar sus saberes técnicos, tecnológicos o profesionales; al atender de forma particular las necesidades y potencialidades de esta población (MEN, 2007).

Para ello, dicha educación permite al adulto mayor ser competitivo en las actividades curriculares al demostrar destrezas, saberes y aprendizajes frente a las demandas, expectativas e intereses de una sociedad cambiante (Bradbury, 2013) y de constantes retos en el proceso de formación (Díez y Flecha, 2010), acaeciendo un mejor nivel personal que se logra mediante prácticas de enseñanza como lo es la andragogía (Ozuah, 2016).

Entendiéndose esta como la disciplina que estudia las estrategias de enseñanza y aprendizaje promoviendo el desarrollo de habilidades y actitudes mediante técnicas, formas, situaciones o procedimientos que permiten un aprender relevante en los adultos (Knowles et al., 2014). Por ello, “... se habla de la adquisición de conocimientos y en la orientación eficaz por parte del docente para lograr las competencias necesarias de enseñanza" (Caraballo, 2007 p.192), pues la educación en adultos es "un proceso de desarrollo integral del ser humano para acceder a la autorrealización, a la transformación propia y del contexto en el cual el individuo se desenvuelve" (Marrero, 2004, p. 7). Lugar donde lo aprendido "se transforma en experiencias aplicables a la vida, no de repetición, sino en una producción de conocimientos de creación, valores, aptitudes, compromiso social y solidario" (Marrero, 2004, p.6).

\section{La alfabetización como garante de equidad y reconocimiento}

Cabe señalar que la educación para adultos que originalmente fue asociada a programas de alfabetización caracterizado por Mendizábal (1990) como un "intento de ayudar al adulto a desarrollar su capacidad de pensar, razonar, expresarse, comprometerse y tomar pastura ante la realidad social, económica y cultural en que vive inmerso" (p. 282), superando la barrera del analfabetismo.

Tal analfabetismo, en este caso particular, aumenta la vulnerabilidad socioeconómica presentando mayores dificultades de inclusión social tanto a nivel personal, familiar y económico (nutrición, higiene, salud y escolaridad de los hijos) como a la sociedad con las pérdidas de productividad y los altos costos para el sistema de salud (Londoño y Ospina, 2016). 
El analfabetismo si bien no está marcado como un problema primordial en la sociedad colombiana (Arias, Gutiérrez y López, 2013), si es un tema que demanda de la atención de la comunidad; puesto que, no saber leer o escribir propende por generar escenarios que dificultan la comunicación y, por ende, el entendimiento (Freire, 2002). En otras palabras, el analfabeta no es un sujeto independiente (Giroux, 2010), siempre necesitara de un tercero para sus procesos de comprensión y producción textual.

Entre tanto, la alfabetización ofrece la posibilidad de acceder a beneficios que, a la par de otras acciones de mejoramiento, elevan su nivel de vida (Giraldo, Zúñiga, Londoño y Sánchez, 2018), además del desarrollo de la actividad creadora donde se inventa y reinventa la realidad en un acto de invención (Muriel, Gómez y Londoño, 2020), con instrumentos que hagan parte tanto del educador, como el educando y del sistema del aprender en el que el hombre toma conciencia de su condición como ser humano despertando sus capacidades e introyectándolas en el conocimiento (Freire, 2002).

\section{Algunos efectos de la educación en el adulto mayor}

Es por esto que el adulto mayor en la educación es motivado por una necesidad que les permite sentirse vivos y útiles para la sociedad (Batalloso, 2006), donde a partir de sus capacidades puedan resolver conflictos, relacionarse con el otro, tener empatía y tomar decisiones oportunas por medio del aprendizaje (Marrero, 2004). Adultos que son "considerados" en la sociedad con una condición difícil a nivel personal, social y familiar para aprender al interior del aula de clases (Caraballo, 2007), ya que pierden habilidades para el trabajo, actividad social y en circunstancias excluidas, cuando en realidad toman la educación con más seriedad, claridad y serenidad porque les permite desarrollar capacidades y habilidades o afianzar las que ya poseen (Mogollón, 2012).

La educación para el desarrollo humano en el adulto mayor presupone una concepción que facilita el proceso de aprendizaje de creencias, hábitos y habilidades trasmitidos de unas personas a otras satisfaciendo las necesidades humanas de vivir y estar seguros, de crear, producir, conocerse y pertenecer (Ospina, 2008), lo cual presupone una visión de la vida, del mundo expresado en la manera de pensar de cada individuo formándolo en la subjetividad e individualidad (Rogers, 2013), siendo el mismo responsable ante el mundo de sus propias capacidades (Nussbaum y Mosquera, 2012), que consisten en la creación de un desarrollo evolutivo, histórico, social y colectivo que da sentido a la vida por medio de la investigación, la narración de cuentos, la 
enseñanza, la discusión y el ejemplo que permiten una enseñanza por medio del aprendizaje (Quintero et al., 2019).

Es así como la educación transforma el territorio (Calvo, 2012), para ello es necesario trascender el aula de clases y generar espacios en la sociedad para que la enseñanza sea el pilar fundamental (Souto-Manning, 2010). Pero ¿Cómo hacerlo?, se puede responder a este interrogante a través de las políticas de cultura ciudadana, de la capacitación, la instrucción, la sensibilización y, en general, aquellos aspectos que permitan la concientización y propendan por la racionabilidad (Suter, 2011). Es necesario darles aplicación a estos preceptos en las esferas, sectores y agremiaciones de la sociedad.

Teniendo en cuenta lo anterior, se puede afirmar que la educación proporciona las herramientas para analizar la realidad donde el hombre va perdiendo sus miedos y se constituye en conciencia de sí mismo y del mundo, en manos que la transforman a través de una pedagogía critica para la formación humana mediante la acción (Freire, 1986). Por tanto, trae consigo la comprensión de la realidad en las prácticas sociales: forma en que se cuenta, modos de hablar, religiosidad, saberes en torno al cuerpo, la sexualidad, la vida, la muerte y la salud (Crichlow, 2013), estas prácticas requieren de una política educacional vivida en las escuelas, lugar en el que se fundan las bases de los contenidos y la participación que abren caminos hacia un mejor aprendizaje (Roberts, 2015); puesto que, educar podría entenderse como la búsqueda realizada por el hombre para alcanzar la comunicación y, al mismo tiempo, con una estrecha relación con la esperanza, adquiriendo un carácter permanente del saber que se aprende desde el mejoramiento constante al aplicar nuevas ideas de vida sin caer en la ignorancia.

Además, porque el adulto mayor debe pretender adaptarse a las posibilidades del quehacer (Allman, 2011), no como proceso de adaptación del estudiante a la sociedad donde hay analfabetismo y desinterés por la educación básica de los adultos (Bracher, 2006), sino de aquel que toma la decisión de crear un canal para un nuevo status, sin seres humanos aislados con la experiencia indispensable de leer la realidad, es decir, de concientizarse de la necesidad de una educación que habla de vocación (Chatier, 2013).

Lo más importante es como el adulto mayor logra compartir espacios comunicativos con la capacidad de comprender, reconocer y reflexionar su entorno por medio de aprendizajes que le ayuden no solo a adquirir conocimientos sino también actuar de forma autónoma tomando acciones sobre ellas (Knowles et al., 2014); logrado al luchar contra los obstáculos que se presentan movilizar y transformar la experiencia partiendo de la realidad que cada 
uno posee, desde la reflexión propositiva hasta las herramientas que permiten la motivación de la enseñanza (Freire, 1986).

Es precisamente, cuando la educación se entiende como un proceso de socialización (Ballantine \& Hammack, 2015) que esta provee al adulto mayor de una conciencia cultural con cambios emocionales, sociales e intelectuales (Arias, et al., 2013), disfrutar de espacios donde se obtiene como herramienta para comenzar el conocimiento, el aprender a conocer (Castillo y Cardona, 2019). En otras palabras, adquirir el conocimiento fomentando la creatividad como formas de expresión en su vivir cotidiano (Martínez de Morentin, 2006). Para llevarlo a cabo es necesario utilizar métodos que permitan respetar las diferencias, desarrollando un juicio propio, activando el pensamiento, encontrando el deseo y demostrando sus capacidades

\section{Conclusiones}

Es esencial partir del reconocimiento a los adultos mayores que, pese a sus circunstancias y entorno, desean estudiar y seguir participando activamente de la vida académica, son un ejemplo para sus familias, las nuevas generaciones y la sociedad en general.

Por otro lado, el concepto de desarrollo humano debe estar presente en los procesos educativos, atendiendo a las realidades de los adultos mayores, del entorno y de los docentes, estableciendo relaciones prácticas y técnicas que construyan conocimiento de una manera efectiva, que otorguen mayores herramientas y preparen de una manera más significativa a dichos educandos.

La educación para los adultos mayores debe apuntar a visualizar y atender las necesidades del territorio, una educación desde el respeto por lo propio, apuntando a tener conciencia del medio ambiente y la sostenibilidad, la cual implicaría un proceso tanto académico como existencial, permitiendo educar para el arraigo y con el objetivo de solucionar las problemáticas del territorio y el empoderamiento de los adultos mayores en él.

Ahora bien, el acompañamiento en el proceso de formación del adulto mayor responde al reconocimiento de las personas que han ayudado a construir nuestro presente; de allí, que sea necesario poder brindarles un lugar de privilegio y de respeto, desdibujando ideas progresistas que apuntan al ciclo vital. 


\section{Referencias}

Alkire, S. (2002). Dimensions of human development. World development, 30(2), 181-205. Recuperado desde https://www.sciencedirect.com/science/article/abs/ pii/S0305750X01001097

Allman, P. (2001). Revolutionary social transformation: Democratic hopes, political possibilities and critical education. Westport: Bergin \& Garvey.

Almiron, M. E. y Porro, S. (2014). Los docentes en la Sociedad de la Información: reconfiguración de roles y nuevas problemáticas. IE Comunicaciones: Revista Iberoamericana de Informática Educativa, (19), 2. Recuperado desde https:// dialnet.unirioja.es/servlet/articulo? codigo $=4794547$

Anderson, R. E. (2008). Implications of the information and knowledge society for education. In International handbook of information technology in primary and secondary education (pp. 5-22). Boston: Springer.

Arias, J. M., Gutiérrez, B. E. y López, R. (2013). Reflexiones sobre alfabetización, educación básica y desarrollo social de personas jóvenes y adultas en Colombia. Revista Interamericana de Educación de Adultos, 35(1), 68-88. Recuperado desde https://www.redalyc.org/pdf/4575/457545115006.pdf

Ballantine, J., \& Hammack, F. M. (2015). The sociology of education: A systematic analysis. London: Routledge.

Barraza, A. (2011). La gestión del estrés académico por parte del orientador educativo.: El papel de las estrategias de afrontamiento. Visión educativa IUNAES, 5(11), 36-44. Recuperado desde http://iunaes.mx/inicio/wp-content/ uploads/2013/04/11-Abril-de-2011.pdf

Batalloso, J.M. (2006). La educación como responsabilidad social. Bases para un nuevo paradigma educativo. Lima: Editorial San Marcos.

Batista, L. (2006). Educación y desarrollo humano. Enfermería en Costa Rica, 27(1), 2830. Recuperado desde http://www.binasss.sa.cr/revistas/enfermeria/v27n1/7.pdf

Bracher, M. (2006). Radical pedagogy: Identity, generativity, and social transformation. New York: Springer.

Bradbury, A. (2013). Education policy and the 'ideal learner': producing recognisable learner-subjects through early years assessment. British Journal of Sociology of Education, 34(1), 1-19. Recuperado desde https://www.tandfonline.com/doi/full/10.1080/01425692.2012.692049?casa_token=wtMMu0lVBuoAAAAA\%3AH3_rleocWKjTfytO_CbwWG14Zh6rIIwXewMji8D4LSqFJfxqfudE9HIKp1NVM35LQA6tRJXJERPoRIcEKOI 
Calvo, C. (2012). Del mapa escolar al territorio educativo. La Serena: Editorial Universidad de La Serena.

Caraballo, R. (2007). La andragogía en la educación superior. Investigacióny Postgrado, 22(2), 187-206. Recuperado desde https://www.redalyc.org/pdf/658/65822208

Castillo, A. y Cardona, S. (2019). El pensar en desarrollo territorial de Caldas con una mirada dialógica/compleja. Latinoamericana de Estudios Educativos, 15(2), 51-85. Recuperado desde http://190.15.17.25/latinoamericana/images/stories/ icono-pdf.jpg

Chartier, R. (2013). Leer la lectura. Trama \& Texturas, (21), 11-23. Recuperado desde https://www.jstor.org/stable/24391623?seq=1

Crichlow, W. (2013). Race, identity, and representation in education. New Yoork: Routledge.

Díez, J. y Flecha, R. (2010). Comunidades de Aprendizaje: un proyecto de transformación social y educativa. Revista interuniversitaria de formación del profesorado, 24(1), 19-30. Recuperado desde https://www.redalyc.org/ pdf/274/27419180002.pdf

Franco, H. A., Londoño, D. A. y Restrepo, D. A. (2017). Habilidades para la vida en jóvenes universitarios: una experiencia investigativa en Antioquia. Katharsis, (24), 155-181. Recuperado desde http://revistas.iue.edu.co/index.php/katharsis/ article/view/970

Freire, P. (1986). La iglesia, la educación y el proceso de liberación humana en la historia. Buenos Aires: La Aurora.

Freire, P. (2002). Concientización: teoría y práctica de una educación liberadora. Ciudad de México: Siglo veintiuno editores. México.

Giraldo, F. L., Zúñiga, S. E., Londoño, D. A. y Sánchez, L. M. (2018). La lectura en la apropiación de la ciencia y la tecnología. Revista Latinoamericana de Estudios Educativos (Colombia), 14(2), 158-178. Recuperado desde https://www.redalyc. org/jatsRepo/1341/134157078008/134157078008.pdf

Giroux, H. A. (2010). Rethinking education as the practice of freedom: Paulo Freire and the promise of critical pedagogy. Policy Futures in Education, 8(6), 715-721. Recuperado desde https://journals.sagepub.com/doi/abs/10.2304/ pfie.2010.8.6.715

Guirao, S. (2015). Utilidad y tipos de revisión de literatura. Ene, 9(2). Recuperado desde http://scielo.isciii.es/scielo.php?script $=$ sci_arttext\&pid $=$ S1988348X2015000200002 
Henao, J. I. y Londoño, D. A. (2017). Relación literacidad, contexto sociocultural y rendimiento académico: la experiencia de la Facultad de Ciencias Empresariales de la Institución Universitaria de Envigado. Encuentros, 15(1), 29-46. doi: 10.15665/ re.v15i1.847.

Knowles, M. S., Holton, E. F., \& Swanson, R. A. (2014). The adult learner: The definitive classic in adult education and human resource development. New York: Routledge.

Lahire, B. (2007). Infancia y adolescencia: de los tiempos de socialización sometidos a constricciones múltiples. Revista de antropología social, 16, 21-37. Recuperado desde https://www.redalyc.org/pdf/838/83811585002.pdf

Londoño, D., y Ospina, H. F. (2016). Capítulo8: La alfabetización crítica: requerimiento social. En Ospina, H. F. y Ramírez-López, C. A. (comps.). Pedagogía crítica latinoamericana y género. pp.235-256. Bogotá: Siglo del Hombre Editores.

López, E., Dominguez, G., Álvarez, F. J. y Jaén, A. (2011). Experiencia didáctica con estudiantes de postgrado sobre los roles del educador en la Sociedad del Conocimiento y la Comunicación con tecnologías 2.0. RELATEC: Revista Latinoamericana de Tecnología Educativa, 10(1), 49-58. Recuperado desde https:// dialnet.unirioja.es/servlet/articulo? codigo $=4160304$

Marrero, T. (2004). Hacia una educación para la emancipación. Núcleo Abierto UNESR.

Martínez de Morentin, J. (2006). ¿Qué es educación de adultos? Responde la Unesco. San Sebastián: Centro UNESCO.

Medrano, L. A., Galleano, C., Galera, M. y del Valle Fernández, R. (2010). Creencias irracionales, rendimiento y deserción académica en ingresantes universitarios. Liberabit, 16(2), 183-192. Recuperado desde http://www.scielo.org.pe/scielo. php?pid=S1729-48272010000200008\&script $=$ sci_arttext\&tlng $=$ en

Mendizábal, M. (1990). La educación básica de adultos. Madrid: Universidad Complutense de Madrid.

Ministerio de Educación Nacional (2007). Definición de educación adulto mayor. Bogotá: Ministerio de Educacion Nacional.

Ministerio de Educación Nacional (2018). Lineamientos generales y orientaciones para la educación formal de personas jóvenes y adultas en Colombia. Bogotá: Ministerio de Educacion Nacional.

Mogollón, E. (2012). Una perspectiva integral del adulto mayor en el contexto de la educación. Revista Interamericana de Educación de Adultos, 34(1), 56-74. Recuperado desde https://www.redalyc.org/pdf/4575/457545090005.pdf 
Muriel, L. E., Gómez, L. S. y Londoño, D. A. (2020). La riqueza de la evaluación en la escuela, un tesoro no oculto. Praxis \& Saber, 11(25), 111-130. Recuperado desde https://revistas.uptc.edu.co/index.php/praxis_saber/article/view/9359

Narro, J., Martuscelli, J. y Barzana, E. (2012). Plan de diez años para desarrollar el Sistema Educativo Nacional. Dirección general de publicaciones y fomento editorial, UNAM. México. Recuperado desde http://www.planeducativonacional.unam.mx

Nussbaum, M. C. y Mosquera, A. S. (2012). Crear capacidades. Barcelona: Paidós.

Ospina,B. (2008). La educación como escenario para eldesarrollohumano. Investigación y Educación en Enfermería, 26(2), 12-15. Recuperado desde http://www.scielo. org.co/scielo.php?script=sci_arttext\&pid=S0120-53072008000300001

Ozuah, P. (2016). First, there was pedagogy and then came andragogy. Einstein journal of Biology and Medicine, 21(2), 83-87. Retrived from http://ojs.library. einstein.yu.edu/index.php/EJBM/article/view/90

Pérez, M., García, R. y Aguaded, I. (2018). Comunicar: calidad, visibilización e impacto. Revista Española de Pedagogía, 76(271), 481-498. Recuperado desde https://www.jstor.org/stable/26547098

Quintero, J., Valencia, Y. P. y Álvarez, D. Y. (2019). Ideais pedagógicos de Paulo Freire na construção de processos de paz, autonomia e democracia nos contextos escolares colombianos. Revista Latinoamericana de Estudios Educativos, 15(2). Recuperado desde http://190.15.17.25/latinoamericana/downloads/ Latinoamericana15(2)_11.pdf

Ramírez, A. (2020). Tendencias investigativas y referentes en los campos de saber: el caso de las líneas de investigación del grupo PAYS. Envigado: Fondo Editorial IUE.

Roberts, P. (2015). Paulo Freire in the 21st century: Education, dialogue, and transformation. London: Routledge.

Rodríguez, Z. E. (2018). Qué y cómo se enseña y aprende en la familia. Un asunto de interés para la escuela. Revista Latinoamericana de Estudios Educativos (Colombia), 14(2), 132-157. Recuperado desde https://www.redalyc.org/ jatsRepo/1341/134157078007/134157078007.pdf

Rogers, C. (2013). Inclusive education and intellectual disability: a sociological engagement with Martha Nussbaum. International Journal of Inclusive Education, 17(9), 988-1002. Recuperado desde https://www.tandfonline.com/doi/ full/10.1080/13603116.2012.727476? casa_token=8RvDs5ADL3IAAAAA\%3A9 OVsatYvSni7NMFuwTlTi77ulk8ZqPVjJxFGeDgWnLF7aH6GCuHy9IVue0YYx85RWF6jnZ0Ee0OPIOG8Cs 
Simkin, H. y Becerra, G. (2013). El proceso de socialización. Apuntes para su exploración en el campo psicosocial. Ciencia, docencia y tecnología, 24(47), 119142. Recuperado desde https://www.redalyc.org/pdf/145/14529884005.pdf

Souto-Manning, M. (2010). Freire, teaching, and learning: Culture circles across contexts. Berlín: Peter Lang.

Suter, W. N. (2011). Introduction to educational research: A critical thinking approach. London: SAGE publications.

Tejada J. y Ferrández, E. (2012). El impacto de la formación continua: claves y problemáticas. Revista Iberoamericana de Educación, 58(3), 1-14. Recuperado desde https://rieoei.org/RIE/article/view/1427

Tuckett, A. (2015). Adult Education, Social Transformation and the Pursuit of Social Justice. European Journal of Education, 50 (3), pp 245-249. Recuperado desde https://wlv.openrepository.com/handle/2436/611265

Ubaldo, S. (2009). Modelo Andragógico. Fundamentos. Ciudad de México: Universidad del Valle de México.

Uribe, M., Ramírez, A. y Londoño, D. (2020). La actualidad de Hermes, el mensajero: De la semiosfera a la ética de la comunicación. En: K. Gherab. Psyché y Polis: Retos socio-político-psicológicos de la educación (9-20). Madrid: Global Knowledge Academics.

Villalobos, J. L., Flórez, G. A. y Londoño, D. A. (2017a). La escuela y la familia en relación con el alcance del logro académico. La experiencia de la Institución Educativa Antonio José de Sucre de Itagüí (Antioquia) 2015. Aletheia. Revista de Desarrollo Humano, Educativo y Social Contemporáneo, 9(1), 58-75. Recuperado desde http:// www.scielo.org.co/scielo.php?pid $=$ S2145-03662017000100058\&script $=$ sci_ abstract\&tlng $=\mathrm{pt}$

Villalobos, J. L., Flórez, G. A. y Londoño, D. A. (2017b). Relación maestro-alcance del logro: Una mirada a los procesos de enseñanza en la escuela. Entramado, 13(1), 186-196. Recuperado desde http://www.scielo.org.co/scielo.php?pid=S190038032017000100186\&script $=$ sci_abstract\&tlng $=$ en 